\title{
SIBERIAN SNAKE FOR THE COOLER SYNCHROTRON COSY
}

\author{
A. Lehrach and R. Maier \\ Forschungszentrum Jülich, 52425 Jülich, Germany
}

\begin{abstract}
Siberian snakes avoid crossing depolarizing resonances in circular accelerators by forcing the spin tune to half integer independent of beam energy. This concept has been proposed by Ya.S. Derbenev and A.M. Kondratenko in 1974 [1]. If only one Siberian snake is used, the invariant spin axis ${ }^{1}$ is in the horizontal plane. This is an interesting feature to deliver longitudinally polarized beam to internal experiments. In this papers possibilities for spin preparation at the Cooler Synchrotron COSY are discussed.
\end{abstract}

\section{INTRODUCTION}

A Siberian snake rotates the spin vectors by $180^{\circ}$ around a horizontal axis (snake axis). The spin vector of the central beam that is invariant after one turn is called the invariant spin axis. The beam polarization is only preserved in this direction. The invariant spin axis is parallel to the snake axis at the symmetry point of the snake, which is located at the opposite side of the snake in the ring. At any other location in the accelerator the invariant spin axis is in the horizontal plan with an orientation depending on the beam energy. It takes two turns for any other spin vector to return to its initial direction, therefore the spin tune is half integer. Transversal or longitudinal fields can be used to build a Siberian snake magnet system. The spin rotation angle $\theta$ in a transversal field $B_{\perp}$ is given by:

$$
\theta=\frac{1+\gamma G}{B \rho} \int B_{\perp} d l,
$$

where $\gamma$ is the Lorentz factor, $G$ the anomalous magnetic moment of the proton, $B$ the bending field and $\rho$ the bending radius. For energies below $10 \mathrm{GeV}$ the orbit deviation of the beam to excite a spin rotation of $180^{\circ}$ in a transversal field is intolerably large. Longitudinal fields $B_{\|}$are only suitable for low beam energies. They have to be increased with beam momentum to keep the spin rotation angle $\theta$ constant during acceleration:

$$
\theta=\frac{1+G}{B \rho} \int B_{\|} d l
$$

In the energy range of COSY a longitudinal field has to be used. The required field strength to build a solenoidal Siberian snake can be calculated using the simple formula: $B_{\|} d l[T m]=3.752 p[\mathrm{GeV} / \mathrm{c}], p$ is the beam momentum.

\section{SPIN PREPARATION}

The COSY injection system only provides vertically polarized protons. With one solenoidal Siberian snake in the

\footnotetext{
${ }^{1}$ Invariant of spin motion for the central beam, called invariant spin field for particle in the six dimensional phase space $[2,3]$.
}

ring the beam has to be injected in a way, that the spin direction of the central beam is longitudinal at the symmetry point of the snake to preserve polarization during the injection process. This can be achieved if the spin is prepared in the injection beam line by additional solenoid magnets. Another possibility is to inject the beam vertically polarized with snake turned off and switch on the snake after injection [4]. The spin vectors follow the invariant spin field without polarization losses if the spin motion is adiabatic. The snake magnet has to be ramped until the spin rotation angle in the snake in $180^{\circ}$. During this process the spin tune of the central beam changes from $\nu_{s p}=\gamma G$ (spin tune without snake) to the nearest half integer tune and depolarizing resonances are crossed (Fig. 1). To avoid

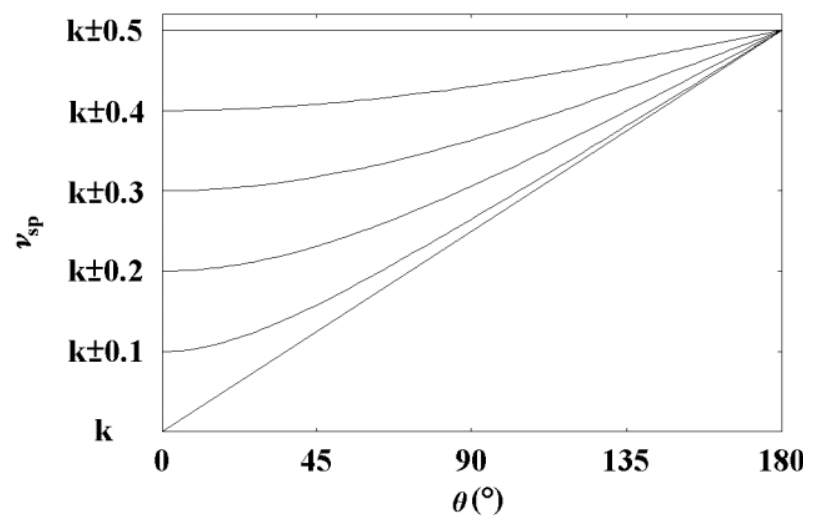

Figure 1: Spin tune $\nu_{s p}$ versus spin rotation angle $\theta, k$ is an integer.

crossing these resonances, the snake can be switch on close to half integer spin tune. If the snake is switched on at half integer spin tune the spin tune stays half integer for any snake strength. This is satisfied whenever the kinetic energy $E_{k i n}$ is given by: $E_{k i n}=370 \mathrm{MeV}+k \cdot 523 \mathrm{MeV}$. In Fig. 2 the spin motion without resonance crossing is shown. As expected, the spin vector changes from vertical direction $\left(S_{y}\right)$ to longitudinal direction $\left(S_{s}\right)$ at the symmetry point of the snake. At injection energy of COSY the spin tune is $\gamma G=1.88$. The spin motion changes rapidly at about 66500 and 72000 turns of the protons after turning on the snake at injection energy (Fig. 3), which leads to polarization losses. The corresponding spin tunes are $\nu_{s p}=1.65$ and $\nu_{s p}=1.63$. Due to the chosen working points in the simulation of $\nu_{x}=3.65$ and $\nu_{y}=3.63$ the two depolarizing resonances $\nu_{s p}=-2+\nu_{x}$ and $\nu_{s p}=-2+\nu_{y}$ are crossed. To reduce these polarization losses the snake has to be ramped slower. Turning on the snake during 250000 turns leads to polarization losses lower than $1 \%$, 


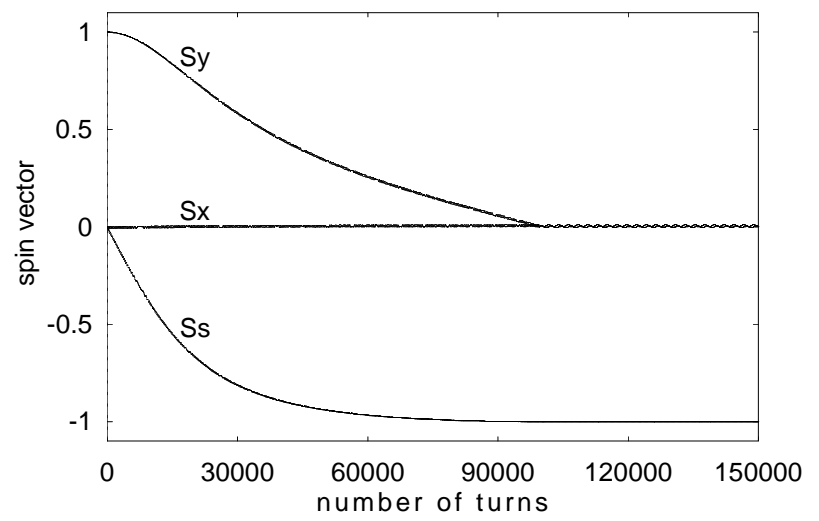

Figure 2: Spin vector $\left(S_{x}, S_{y}, S_{s}\right)$ at the symmetry point of the snake without resonance crossing. The snake is turned on during 100000 turns of the proton in the accelerator.

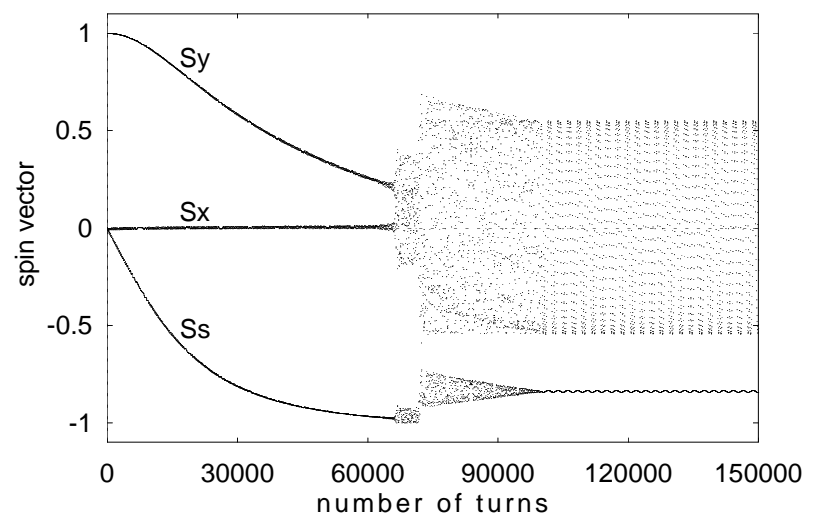

Figure 3: Spin vector at the symmetry point of the snake. The snake is turned on during 100000 turns at injection energy.

as shown in Fig. 4. At injection energy the protons need about $200 \mathrm{~ms}$ for 100000 turns. For normalized transverse beam emittance of $1 \pi \mathrm{mm}$ mrad the degree of preserved polarization versus ramping time of the snake is shown in Fig. 5. At injection energy adiabatic resonance crossing requires a ramping time longer than $0.5 \mathrm{~s}$. The spin vectors are not able to follow the motion of the invariant field if the ramping time is shorter. Very short ramping times in the order of a few milliseconds cannot be reached with the required snake magnet arrangements. Calculations for different energies and transverse beam emittances up to the acceptance limit of COSY indicate that the polarization losses are lower than $1 \%$ for ramping times longer than $1 \mathrm{~s}$.

\section{MAGNET ARRANGEMENT}

A solenoid field up to $12.4 \mathrm{Tm}$ is needed to build a Siberian snake for COSY. Superconducting magnet technology has to be used to achieve an acceptable length of the snake magnet. The solenoid field also rotates the phase space by $32.2^{\circ}$. This can be compensated with two skewed

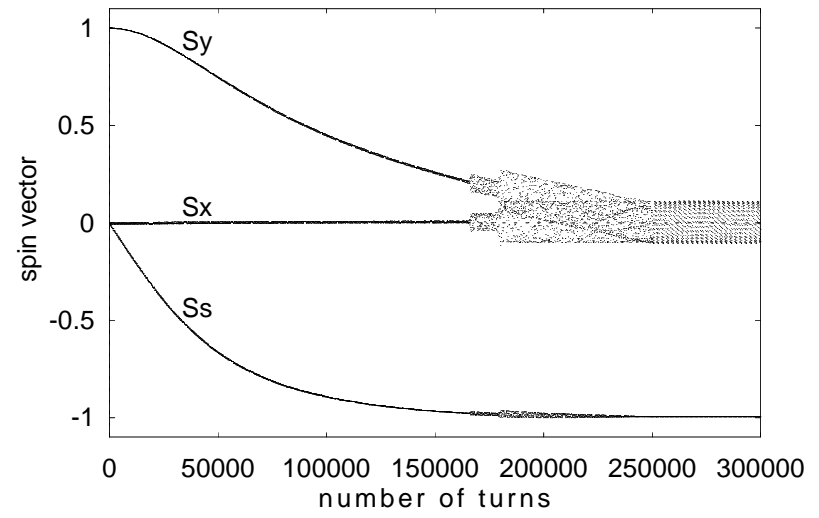

Figure 4: Spin vector at the symmetry point of the snake. The snake is turned on during 250000 turns at injection energy.

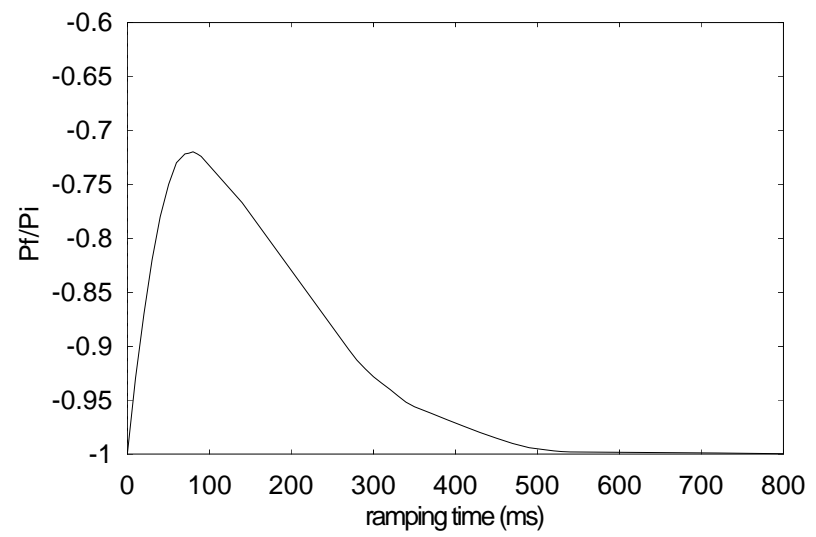

Figure 5: Degree of preserved polarization $\frac{P_{f}}{P_{i}}$ versus ramping time of the snake at injection energy.

quadrupole doublets. Calculations have been done with first order solenoid matrix and quadrupole matrix in thin lense approximation (Fig. 6). Die maximum field strength

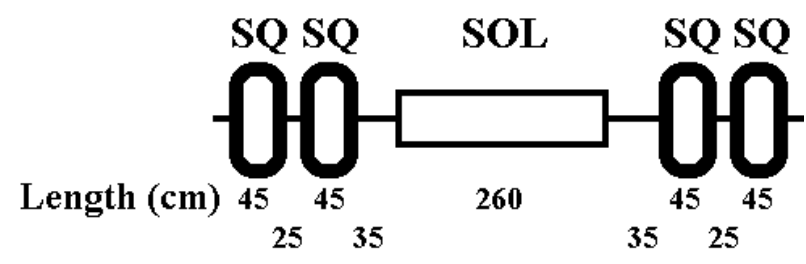

Field (T/m) $34.2-32.2$

Field (T)

$-32.234 .2$

Angle ( $) \quad-21.5-15.2$

4.9

15.221 .5

Figure 6: Magnet arrangement for a Siberian snake consisting of four skewed quadrupole (SQ) and one solenoid magnet (SOL).

of the snake magnet is $4.9 \mathrm{~T}$. The skew quadrupoles are rotated by $21.5^{\circ}$ and $15.2^{\circ}$ with a field gradient of $34.2 \mathrm{~T} / \mathrm{m}$ 
and $-32.2 \mathrm{~T} / \mathrm{m}$. The length of the whole magnet system is $5.6 \mathrm{~m}$ and would fit between quadrupole triplets in the straight sections of COSY.

\section{OPERATION MODES}

There are two different modes to operate the snake:

- The snake can be turned on after injection and ramped during acceleration to avoid crossing depolarizing resonances. Longitudinal polarization is delivered at the symmetry point of the snake for any energy. This mode is suitable for internal experiments which take data during the acceleration cycle of COSY.

- It is also possible to use the snake as a spin rotator. In this case vertically polarized beam has to be accelerated to the desired energy. The snake is turned on after acceleration to transfer the vertical polarization into the horizontal plane and to provide longitudinal polarization at the symmetry point of the snake. Simulations have been done for several energies up to the maximum energy of COSY. This mode can be used for experiments at fixed energy. This operation mode has the advantage of being independent of the accelerator ramping speed.

\section{SPIN PREPARATION FOR EXTRACTED BEAM}

For certain energies a longitudinally polarized beam can also be provided to external experiments, if the snake is turned on during extraction. If one wants to provide longitudinally polarized beam in a large energy range a spin preparation system has to be installed in the extraction beam line. Such a magnet system would consist of two solenoid and two dipole magnets (Fig. 7). For a magnet system with zero effective bending angle of the beam $\left(\alpha_{2}=-\alpha_{4}\right)$ one can show, that the spin rotation angle in the two solenoid magnets to get longitudinal polarization are determined by:

$$
\begin{array}{r}
\cos \alpha_{3}=-\left(\cot \alpha_{2}\right)^{2} \\
\tan \alpha_{1}=\frac{\cot \alpha_{3}}{\cos \alpha_{2}}
\end{array}
$$

The maximum energy range to get longitudinal polarization is restricted by: $\frac{\gamma_{\max }}{\gamma_{\min }}=3$. This energy range can be chosen by varying the bending angle of the beam in the two dipoles. To provide longitudinal polarization up to maximum energy of COSY one has to take a bending angle of $20.6^{\circ}$. The spin rotation angles to get longitudinal polarization for different momenta is listed in table 1 . The maximum integrated field strength of this magnet system is $6.4 \mathrm{Tm}$ and $12.8 \mathrm{Tm}$ in the solenoids and $4 \mathrm{Tm}$ in the dipoles.

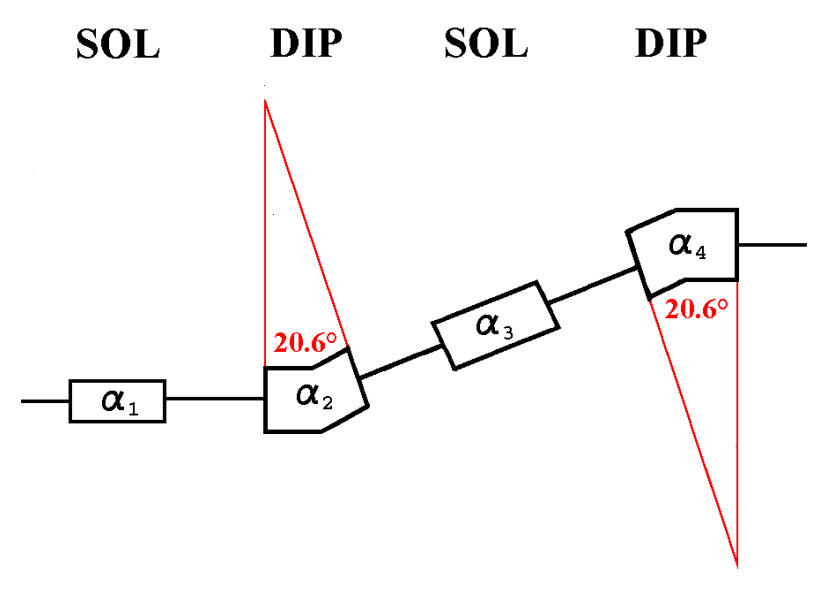

Figure 7: Magnet arrangement for a spin preparation system in the extraction beam line. This magnet system consists of two solenoid (SOL) and two dipole magnets (DIP). The spin rotation angle in the center of mass system of the particles are $\alpha_{1}, \alpha_{3}$ in the solenoids and $\alpha_{2}, \alpha_{4}$ in the dipoles.

Table 1: Spin rotation angles for different momenta to deliver longitudinal polarization in the extraction beam line.

\begin{tabular}{|c|c|c|c|c|}
\hline$p / \frac{\mathrm{GeV}}{c}$ & $\alpha_{1} /^{\circ}$ & $\alpha_{2} /{ }^{\circ}$ & $\alpha_{3} /^{\circ}$ & $\alpha_{4} /^{\circ}$ \\
\hline 0.65 & -90 & 45 & 180 & -45 \\
2.1 & 0 & 90 & 90 & -90 \\
3.3 & 90 & 135 & 180 & -135 \\
\hline
\end{tabular}

\section{CONCLUSION}

Calculations confirm that it is possible to turn on a Siberian snake adiabatically without polarization losses. This method can be used at injection energy to prepare the spin for acceleration with Siberian snake or at higher energies to be able deliver longitudinal polarization for internal experiments. To provide longitudinally polarized beam in a large energy range to external experiments an additional spin preparation system has also to be installed in the extraction beam line.

\section{REFERENCES}

[1] Ya.S. Derbenev and A.M. Kondratenko, Part. Accel. Vol.8, 115 (1978).

[2] Ya.S. Derbenev, A.M. Kondratenko, Sov. Phys. JETP 37(6), 968 (1973).

[3] K. Heinemann and G.H. Hoffstätter et al., Phys. Rev. E 54, 4240 (1996).

[4] W. MacKay et al., 'Commissioning and Future Plans for Polarized Protons in RHIC', these proceedings. 